\title{
Convection in a rotating binary ferrofluid
}

\author{
D. Laroze ${ }^{\mathrm{a}, \mathrm{b}, *}$, J. Martínez-Mardones ${ }^{\mathrm{b}}, \mathrm{J}$. Bragard ${ }^{\mathrm{c}}$, P. $\operatorname{Vargas}^{\mathrm{a}}$ \\ ${ }^{a}$ Departamento de Fisica, Universidad Técnica Federico Santa Maria, Casilla 110-V, Valparaíso, Chile \\ ${ }^{\mathrm{b}}$ Instituto de Física, Universidad Católica de Valparaíso, Casilla 4059, Valparaíso, Chile \\ ${ }^{\mathrm{c}}$ Departamento de Física y Matemática Aplicada, Universidad de Navarra, E-31080 Pamplona, España
}

Available online 19 May 2006

\begin{abstract}
In this work we report theoretical and numerical results on convection for a binary magnetic mixture under rotation. We obtain explicit expressions of convective thresholds in terms of the control parameters of the system for stationary convection. Finally, we analyze the stabilizing effect of rotation on instability thresholds for aqueous suspensions.

(C) 2006 Published by Elsevier B.V.
\end{abstract}

Keywords: Thermal convection; Magnetic fluid; Binary mixture

\section{Introduction}

The development of the nanoscience have permeated trough several scientific areas and technological applications, such as biomedicine or data storage devices. About 30 years ago, de Gennes et al. [1] predicted that colloidal suspension of the small particles with a magnetic dipole moment, ferrofluids, would selfassemble into flexible chains; and today this prediction is an experimental fact, so this behavior of the ferrofluids can be used for further developments of magnetic memory units. Therefore, the theoretical study of the convection and the corresponding instabilities for magnetic nanoparticles in liquid suspensions has relevance in the new directions of the nanotechnology.

Convective instability for a rotating layer of a magnetic fluid have been studied by Gupta et al. [2], and Venkatasubramanian et al. [3]. Recently, Auernhammer et al. [4] formulated the Kuppers-Lortz instability for a magnetic fluid. In addition, Ryskin et al. [5] using the nonequilibrium thermodynamics have derived a complete set of equations to describe ferrofluids in an external magnetic field. They did so in terms of a binary mixture where the magnetophoretic effect, as well as magnetic stresses, have been taken into account in the static and dynamic parts of the ferrofluid equations. In our present work we use the Ryskin approach for including the binary proprieties.

The purpose of this paper is to communicate our analysis of the influence of rotation in convective thresholds in binary magnetic mixtures including the effect of the Soret coefficient for idealized boundary conditions. To this aim, a binary mixture of ferrofluids heated from below and rotated around the vertical axis

\footnotetext{
*Corresponding author. Departamento de Física, Universidad Técnica Federico Santa María, Casilla 110-V, Valparaiso, Chile. Tel.: + 5632654501 ; fax: + 5632787656 .

E-mail address: david.laroze@usm.cl (D. Laroze).
} 
is considered. The description of this system involves many parameters whose values differs in many order of magnitude; so as a fist step in understanding the different convection regimes, we do not take into the account the magnetophoretic effect and we consider a simplified balance equation for magnetic nanoparticle concentration, that is, without a complete coupling to the magnetic field. The linear stability analysis of the conduction state is performed and we find an analytical expression of the Rayleigh number.

\section{Basic equations}

We consider a layer of incompressible binary magnetic fluid, of thickness $d$, parallel to the $x y$-plane, with very large horizontal extension, in a gravitational field $\mathbf{g}$ and submitted to a vertical temperature gradient. The layer is rotating uniformly about the vertical with uniform angular velocity $\boldsymbol{\varpi}$. The magnetic fluid properties can be modeled as electrically nonconducting superparamagnets, and it is assumed to be placed in a magnetic field $\mathbf{H}$ parallel to $\hat{\mathbf{z}}$, which would be homogeneous if the magnetic fluid were absent. Let us choose the $z$-axis such that $\mathbf{g}=-g \hat{\mathbf{z}}$ and that the layer has its interfaces at $z=0$ and $z=d$. A static temperature difference across the layer is imposed, $T(z=0)=T_{0}+\triangle T$ and $T(z=d)=T_{0}$. Under the Boussinesq approximation, the dimensionless balance perturbation of the conduction states and the Maxwell equations read as [5]

$$
\begin{aligned}
& \nabla \cdot \mathbf{v}=\mathbf{0} \\
& \left(\partial_{t}+\mathbf{v} \cdot \nabla\right)\left(\theta-M_{4} \partial_{z} \phi\right)=R_{a}\left(1-M_{4}\right) w+\nabla^{2} \theta, \\
& \left(\partial_{t}+\mathbf{v} \cdot \nabla\right) c=R_{a} w+L \nabla^{2}(c-\theta), \\
& P^{-1}\left(\partial_{t}+\mathbf{v} \cdot \nabla\right) \mathbf{v}=-\nabla p_{e f f}+\nabla^{2} \mathbf{v}+T_{a}^{1 / 2} \mathbf{v} \times \hat{\mathbf{z}} \\
& \left.+\left\{\left(1+M_{1}\right) \theta+\psi c\right)-\left(M_{5}-M_{1}\right) \partial_{z} \phi\right\} \hat{\mathbf{z}}+M_{1} \theta \nabla\left(\partial_{z} \phi\right), \\
& \left(\nabla^{2}+M_{3} \nabla_{\perp}^{2}\right) \phi-\partial_{z} \theta=0, \\
& \nabla^{2} \phi_{\text {ext }}=0,
\end{aligned}
$$

where $\mathbf{v}=(u, v, w)^{\mathrm{T}}$ is the velocity field, $\theta$ is the temperature, $p_{\text {eff }}$ is the effective pressure, $\phi$ and $\phi_{\text {ext }}$ are the internal and the external scalar magnetic potential, respectively. Also the following groups of dimensionless numbers have been introduced: (a) (pure fluids) the Rayleigh number $R_{a}=\alpha_{T} g \triangle T d^{3} / \kappa v$ accounting for buoyancy effects and the Prandtl number $P=v / \kappa$, relating viscous and thermal effects; (b) (rotation in pure fluids) the Taylor number $T_{a}=\left(2 \varpi d^{2} / v\right)^{2}$; (c) (binary mixtures) the Lewis number $L=D / \kappa$, relating diffusion with thermal diffusivity, and the separation ratio $\psi=\alpha_{c} k_{T} / \alpha_{T} T_{0}$; and (d) (magnetic fluid) the strength of magnetic force relative to buoyancy $M_{1}=\mu_{0} \kappa^{2} \triangle T /\left(d g \alpha_{T} \rho_{0}\left(1+\chi_{H}\right)\right)$, the nonlinearity of magnetization $M_{3}=\left(1+M_{0} / H_{0}\right) /\left(1+\chi_{H}\right)$ is a measure of the deviation of the magnetization curve from the linear behavior $M_{0}=\chi_{H} H_{0}$, the the magnetophoretic number $M_{4}=\mu_{0} T_{0} \kappa^{2} / c_{0} \rho_{0}\left(1+\chi_{H}\right)$ which gives rise to a field dependence of heat and concentration currents, and the ratio of magnetic to thermal buoyancy $M_{5}=\alpha_{H} \chi_{T} H_{0}^{2} /\left(\alpha_{T}\left(1+\chi_{H}\right)\right)$. Let us comment about of the numerical values of the parameters, the parameters $R_{a}$ and $T_{a}$ may be changed in several orders of magnitude. A typical value for $P$ is $P \approx 10$ (water). For aqueous suspensions of ferrofluid, $L \approx 10^{-4}$ and the separation ratio $\psi$ is taken positive, so there is only the stationary convection case. The magnetic numbers have the following order of magnitude $M_{1}=10^{-4}-10^{1}$, $M_{3} \approx 1.1, M_{4} \approx M_{5} \approx 10^{-6}$ [4,5]. The values of $M_{4}$ and $M_{5}$ are very small, so in our calculation we will not take them into account neither, for our analysis, is necessary to use the external scalar magnetic potential, $\phi_{\text {ext }}$. In the next section, we study the corresponding linear stability analysis of the conduction state.

\section{Results}

For calculating the linear stability analysis, we only need the linear parts of Eqs. (1)-(5), the effective pressure and two components of the velocity field could easily be eliminated by applying the rotor and double rotor operator in the Navier-Stokes equation and then considering only the $z$-component of the resulting 

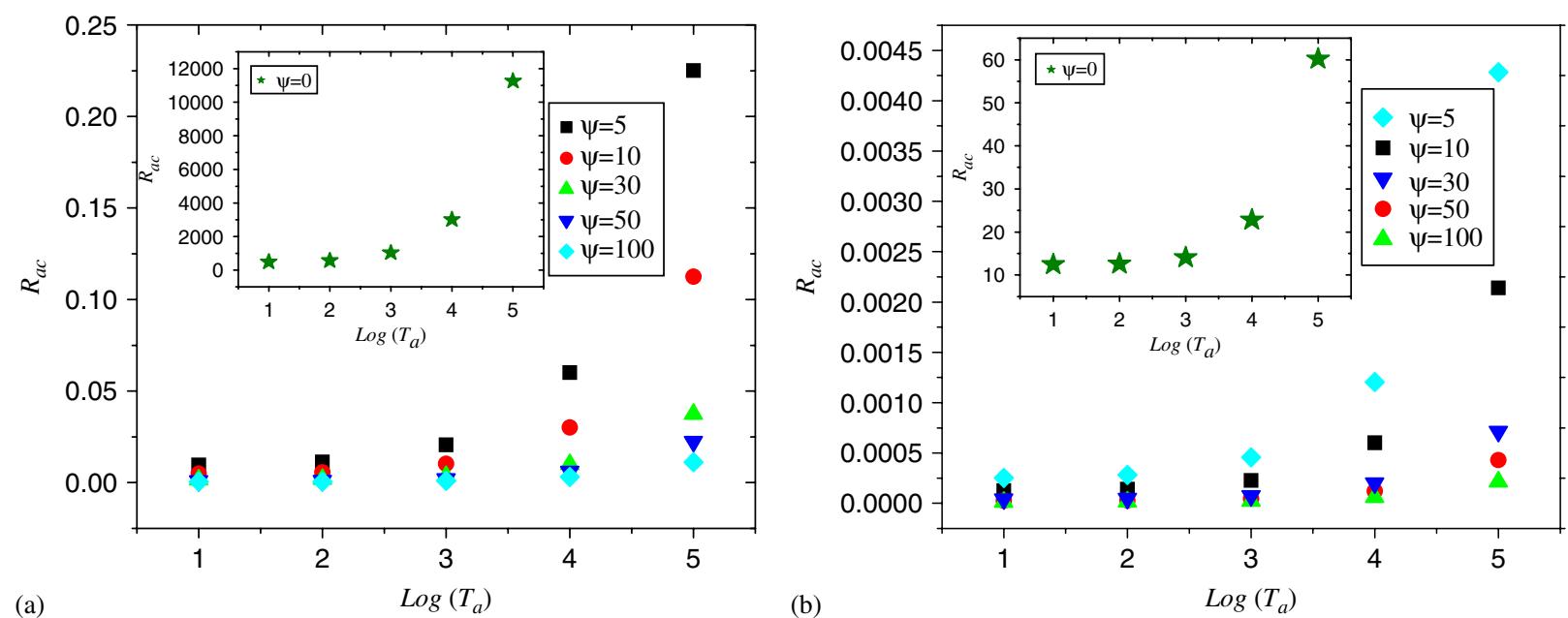

Fig. 1. Critical Rayleigh number $R_{a c}$ as function $T_{a}$ for $M_{1}=1$ (a) and for $M_{1}=10$ (b) at different values of $\psi$. The insets are for $\psi=0$.

equations. To solve the resulting system of equations and then to find analytical expressions, the idealized boundary conditions: $w=\partial_{z}^{2} w=\theta=\eta=\partial_{z} \phi=0$, at both $z=0$ and 1 are considered; so with them the eigenvalue problem gives the Rayleigh number. The stationary bifurcation is obtained for the eigenvalue equal to zero and for the fundamental mode, gives the marginal curve for stationary convection:

$$
R_{a}=\frac{\left(\pi^{2}+k^{2} M_{3}\right)\left(\pi^{2} T_{a}+\left(\pi^{2}+k^{2}\right)^{3}\right)}{k^{2}\left[\left(1+M_{1}\right)\left(\pi^{2}+k^{2} M_{3}\right)-\pi^{2} k^{2} M_{1}\right]\left(1+\psi\left(1+L^{-1}\right)\right)} .
$$

Note that, Eq. (7) reproduce all the know result, for example, if we take $\psi=0$ we obtain the expression of $R_{a}$ for a simple ferrofluid [4]. The minimum of the marginal curve $\left(\partial_{k} R_{a}=0\right)$ gives the critical wavenumber $k_{c}$ and, the critical Rayleigh number, $R_{a c}$, can be obtained by replacing it in Eq. (7). The main results are displayed in Fig. 1, where we plot the critical Rayleigh number, $R_{a c}$, as function of the Taylor number, $T_{a}$, for different values of the separation ratio $\psi$ at $P=10, L=10^{-4}, M_{3}=1.1$; two different values of the strength of magnetic force relative to buoyancy are considered: $M_{1}=1$ (Fig. 1(a)) and $M_{1}=10$ (Fig. 1(b)). The corresponding threshold value decreases as $\psi$ increases, while it increases when $T_{a}$ increases, so the rotation rate is a stabilizing effect; and it is weaker when $M_{1}$ increases. We have found, for these fixed parameters, that $\psi_{b} R_{a c}\left(\psi_{a}\right)=\psi_{a} R_{a c}\left(\psi_{b}\right)$. Furthermore, for the chosen range of $L$ and $\psi$, we can very good approximate the Rayleigh number of Eq. (7) by $R(\psi, L, x) \approx(L / \psi) R_{r 0}(x)$, where $R_{r 0}$ is the Rayleigh number for a simple rotating ferrofluid; this implies that the binary effects are destabilizing effects and $R_{a c}$ is hugely reduced respect to $R_{r o c}$ as shown in the inset of both frames.

\section{Final remarks}

In the present work, Rayleigh-Benard convection in a binary magnetic fluid liquid mixture under rotation is studied. We determine the stability thresholds for stationary convection for idealized boundary conditions. These results suggest that a rotation can be used as a tool to enhance the range of the laminar regime of convective in aqueous solutions. Beside, the binary effects drastically reduce the critical Rayleigh number. We will deal with more realistic rigid-rigid boundary conditions in a future work.

\section{Acknowledgements}

We emotionally dedicate this article to professor Carlos Perez-Garcia (Q.E.D.) for his motivations and interchange of ideas about Hydrodynamics Instability. This research received financial support from CONICYT Ph.D. program fellowship and Millennium Science Initiative, Chile. 


\section{References}

[1] P.G. de Gennes, P.A. Pincus, Phys. Kondens. Mater. 11 (1970) 189.

[2] M.D. Gupta, A.S. Gupta, Int. J. Eng. Sci. 17 (1979) 271.

[3] S. Venkatasubramanian, P.N. Kaloni, Int. J. Eng. Sci. 32 (1994) 237.

[4] G.K. Auernhammera, H.R. Brand, Eur. Phys. J. B 16 (2000) 157.

[5] A. Ryskin, H. Pleiner, Phys. Rev. E 69 (2004) 046301. 